\title{
Aerodynamic study of three cars in tandem using computational fluid dynamics
}

\author{
H. Abdul-Rahman ${ }^{1}$, H. Moria² and M.R. Rasani1 \\ 1 Department of Mechanical and Manufacturing Engineering, Faculty of Engineering and Built Environment, Universiti Kebangsaan Malaysia, \\ 43600 Bangi, Selangor, Malaysia \\ Phone: +60389216522; Fax: +60389118314 \\ ${ }^{2}$ Department of Mechanical Engineering Technology, Yanbu Industrial College, Yanbu Al-Sinaiyah City 41912, Saudi Arabia
}

\begin{abstract}
Aerodynamics of vehicles account for nearly $80 \%$ of fuel losses on the road. Today, the use of the Intelligent Transport System (ITS) allows vehicles to be guided at a distance close to each other and has been shown to help reduce the drag coefficients of the vehicles involved. In this article, the aim is to investigate the effect of distances between a three car platoons, to their drag and lift coefficients, using computational fluid dynamics. To that end, a computational fluid dynamics (CFD) simulation was first performed on a single case and platoon of two Ahmed car models using the STAR-CCM+ software, for validation with previous experimental studies. Significant drop in drag coefficients were observed on platoon models compared to a single model. Comparison between the $k-\omega$ and $k-\varepsilon$ turbulence models for a two car platoon found that the $k-\omega$ model more closely approximate the experimental results with errors of only $8.66 \%$ compared to $21.14 \%$ by $k-\varepsilon$ turbulence model. Further studies were undertaken to study the effects of various car gaps $(0.5 L, 1.0 L$ and $1.5 L ; L=$ length of the car) to the aerodynamics of a three-car platoon using CFD simulation. Simulation results show that the lowest drag coefficient that impacts on vehicle fuel savings varies depending on the car's position. For the front car, the lowest drag coefficient $\left(C_{D}\right)$ can be seen for car gaps corresponding to $X_{1}=0.5 \mathrm{~L}$ and $X_{2}=0.5 \mathrm{~L}$, where $C_{D}=$ 0.1217 , while its lift coefficient $\left(C_{L}\right)$ was 0.0366 ( $X_{1}$ and $X_{2}$ denoting first to second and second to third car distance respectively). For the middle car, the lowest drag coefficient occurred when $X_{1}=$ $1.5 \mathrm{~L}$ and $X_{2}=0.5 \mathrm{~L}$, which is 0.1397 . The lift coefficient for this car was -0.0611 . Meanwhile, for the last car, the lowest drag coefficient was observed when $X_{1}=0.5 L$ and $X_{2}=1.5 L$, i.e. $C_{D}=0.263$. The lift coefficient for this car was 0.0452 . In this study, the lowest drag coefficient yields the lowest lift coefficient. The study also found that for even $X_{1}$ and $X_{2}$ spacings, the drag coefficient increased steadily from the front to the last car, while the use of different spacings were found to decrease drag coefficient of the rear car compared to the front car and had a positive impact on platoon driving and fuel-saving.
\end{abstract}

ARTICLE HISTORY

Received: $24^{\text {th }}$ July 2020

Revised: 24 $4^{\text {th }}$ Apr 2021

Accepted: $02^{\text {nd }}$ June 2021

\section{KEYWORDS}

Computational fluid

dynamics (CFD);

platoon;

aerodynamic force;

drag coefficient.

\section{INTRODUCTION}

With the increasing number of vehicles on the road today, the concept of platoon was introduced to improve traffic efficiency [1-3]. According to Horowitz [4], a platoon involves organizing a group of nearby vehicles and may help to increase road capacity. Vehicles will be able to travel 30 feet or less by using standard distance sensors in most cars. The concept of platoon also provides dramatic reduction in fuel consumption at highway speeds through reduced aerodynamic load on vehicles [5-7]. According to Hucho [8], aerodynamic loads on standard-sized cars moving at $62 \mathrm{mph}(100 \mathrm{~km} / \mathrm{h})$ contribute to almost $75-80 \%$ of vehicle fuel losses. Hence, it is relevant to examine the effect of distance between vehicles in the platoon towards the reduction of drag and lift forces, for more efficient fuel consumption.

A number of studies have been conducted by various organizations in relation to the effect of distance on the aerodynamic forces of platoon vehicles [9-12]. An on-the-road experiment performed by California Partners for Advanced Transit and Highways (PATH) using heavy trucks equipped with Intelligent Transport System (ITS), had demonstrated technical feasibility for the driving of two trucks with a $3 \mathrm{~m}$ gap and three trucks with $4 \mathrm{~m}$ gaps. The results showed direct fuel saving of within $5 \%$ for the main truck and 10-15\% for the following trucks [12]. Effective energy saving observed from the PATH project was associated with aerodynamic drag reduction due to reduced distances between vehicles.

Previous studies of platoon vehicles in excess of two vehicles (ie. three vehicles or more) had involved uniform distances between vehicles as reviewed by Rajamani [13], Schito and Braghin [14] and Davila et al. [5]. Therefore, it would be interesting to know the impact of different gaps between vehicles towards aerodynamic forces of each vehicle involved in the platoon. Furthermore, only a small number of studies have studied the relationship between drag and lift to each car in a platoon and this phenomenon has not been fully understood. With high-speed cars being a common phenomenon today, reducing the coefficient of lift to improve stability on the road is no longer a concern for racing cars 
alone [15]. A previous study indicated that platoons with large number of vehicles may not be practical due to instabilities and traffic conditions [16], and as a result, we limit our attention in the present study to a three vehicle platoon.

Currently, computational fluid dynamics (CFD) simulations have been widely used in place of actual experiments for analyzing the flow of air around a body due to their improved processing time and ability to analyze various situations or conditions that are not practical by experiments. Not surprisingly, the overall validation of fluid simulation is dependent on the turbulent model and meshing scheme applied [17,18]. For the aerodynamic simulation study of vehicles using CFD, the two RANS (Reynolds-Averaged Navier Stokes) models frequently used are the $k$-epsilon $(k$ - $\varepsilon$ ) and $k$-omega $(k$ $\omega)$ models. Nevertheless, the type of model that provides the most accurate and time-saving solution for the case of platoon vehicles is unknown. Hence, it is important to compare the simulation between $k-\varepsilon$ and $k-\omega$ models to identify the appropriate turbulent model in terms of accuracy and computational efficiency for this study.

In the present study, the Ahmed car model based on the study of Ahmed et al. [19] has been used. An Ahmed body or model is basically a simplified car model with a rear slant [20]. The Ahmed model is composed of a flat but roundededge forward section, a removable slant section placed at the rear of the body to study separation phenomena at different rear inclination angles, and a rectangular box that connects the front and rear inclination angles [21]. It is widely used to represent the shape of a real car because of its simple geometry for modeling, and the ability to emulate the typical car airflow especially sedan-shaped cars as in studies by Bayraktar et al. [22], Minguez et al. [23], Bruneau et al. [24], Serre et al. [25] and Madharia et al. [26]. Figure 1 shows an actual model of an Ahmed car. In this study, we considered a rear slant angle of $30^{\circ}$, which replicates drag coefficients and typical slant angle of present cars.

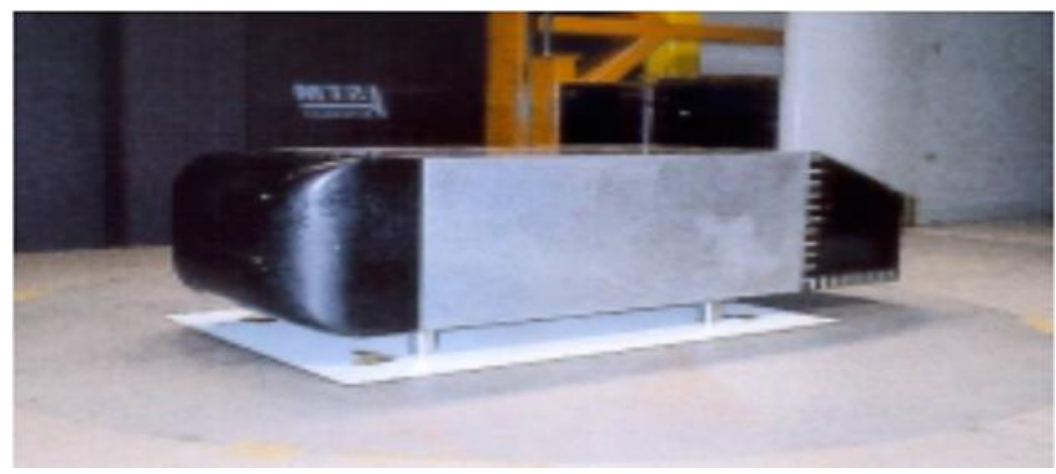

Figure 1. An Ahmed car model [21]

\section{METHODS AND MATERIALS}

\section{Geometry and Flow Domain}

The present aerodynamic studies of three cars in tandem using computational fluid dynamics had involved two main phases. The first phase involved the construction of Ahmed's car model geometry and fluid domain using CAD Inventor, while STAR-CCM+ software was used to run aerodynamic simulations on the car model. Figure 2 presents the geometry of the Ahmed car model and computational flow domain used in the numerical simulations. The length $(L)$, width $(W)$ and height $(H)$ of the Ahmed body are $1044 \mathrm{~mm} \times 389 \mathrm{~mm} \times 288 \mathrm{~mm}$ respectively $[19,20]$.

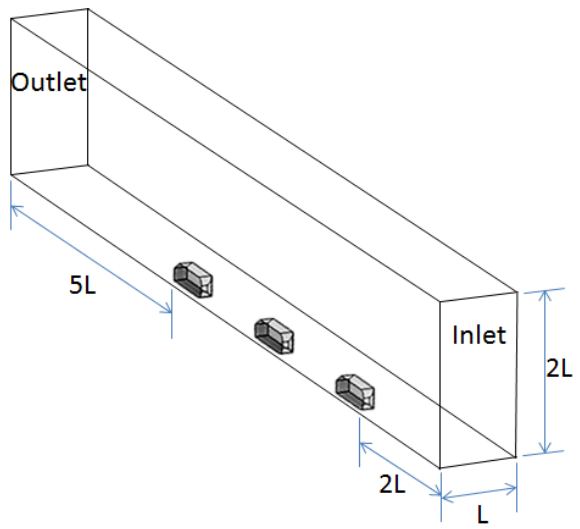

(a)

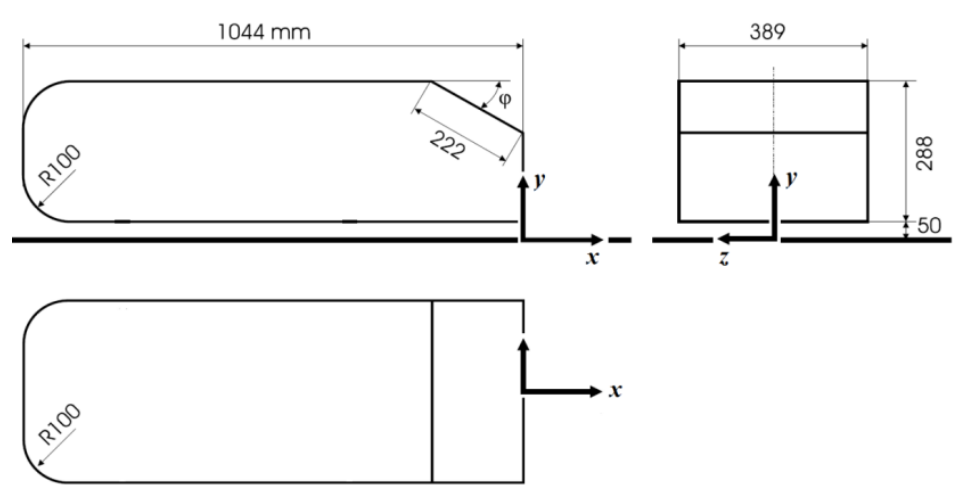

(b)

Figure 2. (a) Computational domain and (b) Geometry of Ahmed car model (courtesy of [19,20]) 
The streamwise (ie. $x$-direction) dimension of the flow domain was set such that the distance from the front of the first body to the inlet is $2 L$ and the distance from the rear of the last body to the outlet is $5 L$. While the spanwise (ie. $z$-direction) and height (ie. $y$-direction) of the flow domain were set as $1 L \times 2 L$ respectively, giving a blockage ratio of approximately $5.1 \%$, which is within the recommended $10 \%$ limit where blockage effect may not be negligible [27].

A total of nine cases were studied that involved a gap of $0.5 \mathrm{~L}, 1.0 \mathrm{~L}$ and $1.5 \mathrm{~L}$ between the cars. The study was limited to maximum spacing of $1.5 \mathrm{~L}$ based on a research by Watkins and Vino [28], which found no significant change between the drag coefficient of a car in a platoon and a single car when exceeding such distance. $X_{l}$ represents the distance between car 1 and car 2 while the $X_{2}$ represents the distance of car 2 to car 3 . Figure 3 shows the side view of car positions for simulation purposes.

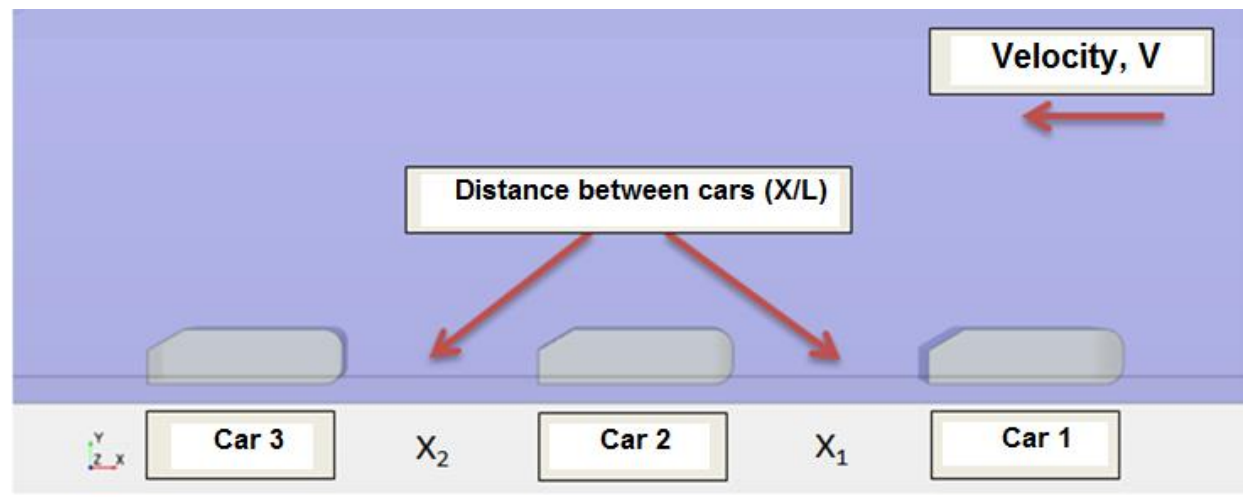

Figure 3. The position of the car and their distances for the simulation

\section{Flow Governing Equations}

The aerodynamics around the cars was captured using both the conservation of momentum and conservation of mass in the flow. For incompressible and Newtonian fluid, these correspond to the Navier-Stokes and continuity equations respectively. As the flow and wakes anticipated in this problem may possibly be unsteady and turbulent, we considered the unsteady Reynolds-Averaged Navier Stokes (RANS) turbulent models, which use the following form for the NavierStokes and continuity equations:

$$
\begin{gathered}
\rho\left(\frac{\partial \bar{u}_{i}}{\partial t}+\bar{u}_{j} \frac{\partial \bar{u}_{i}}{\partial x_{j}}\right)=-\frac{\partial \bar{p}}{\partial x_{i}}+\frac{\partial}{\partial x_{j}}\left\{\mu_{e f f}\left(\frac{\partial \bar{u}_{i}}{\partial x_{j}}+\frac{\partial \bar{u}_{j}}{\partial x_{i}}\right)\right\} \\
\frac{\partial \bar{u}_{i}}{\partial x_{i}}=0
\end{gathered}
$$

where $\bar{p}$ and $\bar{u}_{i}$ denotes the time-averaged pressure and velocity components respectively $(i=1,2,3$ for three-dimensional problems). In addition, $\mu_{\text {eff }}=\mu+\mu_{t}$ represents the effective viscosity in the fluid considering its dynamic viscosity $(\mu)$ and viscosity due to turbulent effects $\left(\mu_{t}\right)$ - which needs to be determined using the RANS turbulence model used. In the present problem, standard air properties are used, where its constant density $(\rho)$ is $1.225 \mathrm{~kg} / \mathrm{m}^{3}$ and its dynamic viscosity $(\mu)$ is $1.856 \times 10^{-5} \mathrm{~kg} /(\mathrm{m} . \mathrm{s})$.

For the selection of turbulent models, numerical experiments will be conducted on two types of RANS turbulent models available in STAR-CCM + to identify model with the least error compared to experimental results, which are:

1. Realizable $k-\varepsilon$ : The $k-\varepsilon$ model solves two variables: $k$ (ie. turbulence kinetic energy) and $\varepsilon$ (ie. turbulence kinetic energy dissipation rate). Turbulent $k-\varepsilon$ model is very popular for industrial applications due to their good convergence rates and low memory requirements. It does not accurately model flow regions with adverse pressure gradients or strong curvature for flow, or a jet stream, but is useful for external flow problems around complex geometries. Realizable $k-\varepsilon$ is an improvement from the standard $k-\varepsilon$ [29]. This model contains a new formula for turbulent viscosity $\left(\mu_{t}\right)$ and new transport equations for dissipation rates $\varepsilon$.

2. SST (Menter) $k$ - $\omega$ : The $k$ - $\omega$ model is similar to the $k-\varepsilon$ model, but instead of solving the dissipation rate $\varepsilon$, it solves for $\omega$ (ie. specific turbulence kinetic energy dissipation rate). It also uses wall functions and therefore has comparable memory requirements. It has more difficulty converging and is quite sensitive to the initial guess of the solution. Therefore, $k-\varepsilon$ model is often used first to find the initial state to solve the $k$ - $\omega$ model. This model is useful in many cases where the $k-\varepsilon$ model is inaccurate, such as internal flow, flow that shows strong curvature, separated flows and jets. The shear stress transport (SST) model is a variant of the standard $k-\omega$ model that 
utilizes a blending function, allowing it to behave like a $k$ - $\omega$ model in the region near the wall and like a $k$ - $\varepsilon$ model in the free flow region of the fluid domain.

\section{Boundary Conditions}

Figure 4 shows the boundary types prescribed on all the boundaries in the computational domain and Table 1 summarizes their corresponding boundary conditions employed in the present study.

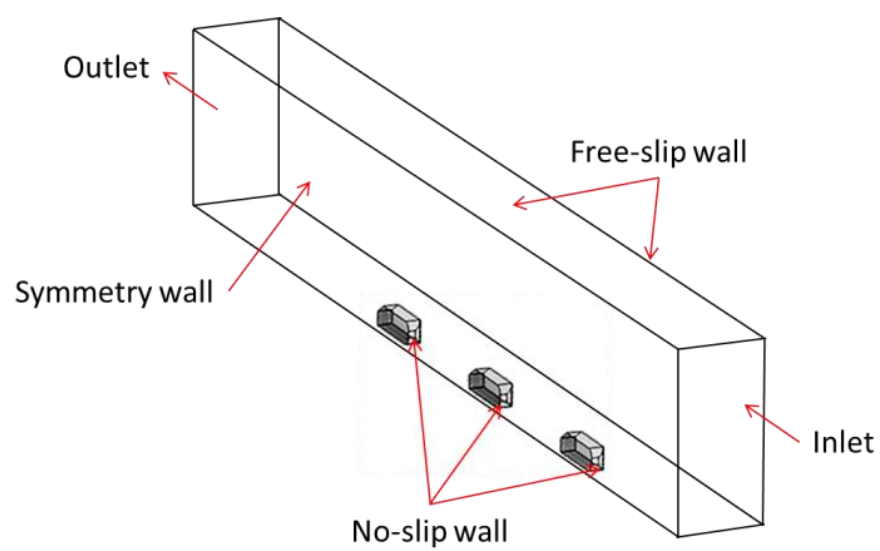

Figure 4. Boundary conditions defined in the flow domain

Table 1. Boundary conditions prescribed in the present model

\begin{tabular}{|c|c|c|}
\hline Boundary & $\begin{array}{l}\text { Conditions } \\
\text { Prescribed }\end{array}$ & Input Parameters \\
\hline \multirow{2}{*}{ Inlet } & Velocity inlet & $\begin{array}{c}U=25 \mathrm{~m} / \mathrm{s}, \mathrm{v}=0, \mathrm{w}=0 \text { (for case studies) } \\
U=35 \mathrm{~m} / \mathrm{s}, \mathrm{v}=0, \mathrm{w}=0 \text { (for validation studies) }\end{array}$ \\
\hline & $\begin{array}{l}\text { Turbulent } \\
\text { intensity }\end{array}$ & $1.8 \%$ \\
\hline Outlet & Pressure outlet & $0 \mathrm{~Pa}$ (relative atm. pressure) \\
\hline Left face & Symmetry & \\
\hline Car faces & Wall & No-slip \\
\hline $\begin{array}{l}\text { Top, right, } \\
\text { bottom face }\end{array}$ & Wall & Free-slip \\
\hline
\end{tabular}

\section{Numerical Grid Generation}

In order to solve the governing flow equations, the flow domain is discretized into smaller elements, allowing appropriate numerical schemes to be used. Figure 5 samples the meshing scheme tested and adopted in the present study. In general, a mesh control volume was used where finer grids are prescribed in regions close to the cars to accurately resolve the boundary layers, while coarser grids are generated away from the cars. This also includes finer grids behind and in between the cars to increase flow resolution in the wake regions of the cars.

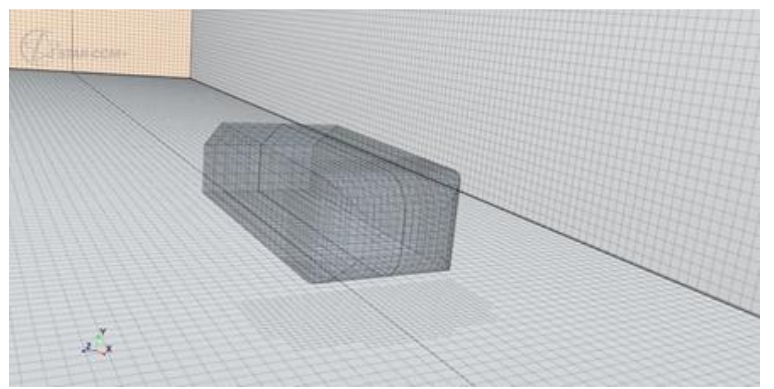

(a)

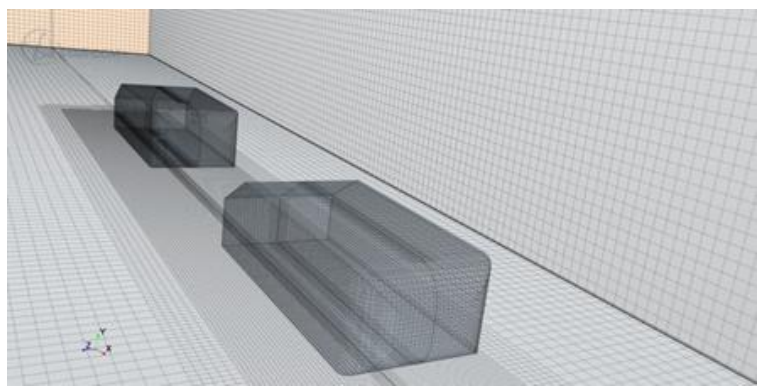

(b)

Figure 5. Meshing scheme employed: (a) single car (b) two-car platoon 
To obtain accurate and time-saving simulation results, mesh independence studies were first conducted, involving comparisons with experimental results by Ahmed et al. [19] for the case of a single car and by Watkins and Vino [28] for a two-car platoon. In the first case involving a single car, it was found that a mesh with a total 230818 elements was sufficient to get results approximating the experimental value. Table 2 shows the drag coefficient comparison between the two types of turbulent models $(k-\varepsilon$ and $k-\omega)$ involving a single car.

Table 2. Comparison of drag coefficients for a single car

\begin{tabular}{|c|c|c|}
\hline Turbulence model & $\begin{array}{c}\text { Drag coefficient } \\
C_{D}\end{array}$ & $\begin{array}{c}\text { Error } \\
\mathrm{C}_{\mathrm{D}}=0.378[19]\end{array}$ \\
\hline$k-\omega$ & 0.376 & $-0.56 \%$ \\
\hline$k-\varepsilon$ & 0.375 & $-0.88 \%$ \\
\hline
\end{tabular}

Based on the results of the study, both turbulent models produced very low error values. However, the $k$ - $\omega$ yielded closer drag coefficient value with the experiment (ie. 0.376 ) with only $-0.56 \%$ error compared to $k-\varepsilon$ with error $0.88 \%$. Taking into account the accuracy of $k-\omega$ in obtaining the drag coefficient values closest to the experiment, the mesh-independence study was continued using this turbulent model for two Ahmed body in a platoon arrangement (with gap $1 L$ ) and compared to the results of the wind tunnel experiments by Watkins and Vino [28]. Inlet velocity was set at $35 \mathrm{~m} / \mathrm{s}$ with time-step of $0.001 \mathrm{~s}$. Table 3 shows the comparison of the drag coefficient results for three different mesh sizes for the rear car - one of which considers no volume control around the car (where a volume control prescribes finer grids inside a volume surrounding or close to the bodies, to increase flow resolution in this region).

Table 3. Mesh-independence study of two-car platoon

\begin{tabular}{cccc}
\hline Total mesh & $\begin{array}{c}\text { Volume Control } \\
\text { Around Car }\end{array}$ & $\begin{array}{c}\text { Drag coefficient } \\
\mathrm{C}_{\mathrm{D}}\end{array}$ & $\begin{array}{c}\text { Error } \\
\mathrm{C}_{\mathrm{D}}=0.25[28]\end{array}$ \\
\hline 518435 & No & 0.312 & $19.87 \%$ \\
724974 & Yes & 0.274 & $8.66 \%$ \\
938092 & Yes & 0.275 & $9.22 \%$ \\
\hline
\end{tabular}

Based on the table above, it can be seen that when volume control is not applied around the car, i.e. with total 518435 elements, the drag coefficient obtained is significantly different than the value obtained from the experimental results, with error of $19.87 \%$. When volume control is applied around the car, the mesh total increased to 724974 elements. The drag coefficient is approaching experimental value with an error of only $8.66 \%$. The decline in this error is due to finer mesh detail in the rear car wake region that affects the value of the drag coefficient. The mesh is then increased to 938092 elements to further refine meshing around the car. However, the value of the drag coefficient obtained is not significantly different from the previous mesh configuration. Hence, for the purposes of accuracy and time-saving simulation, the mesh model that produces 724974 elements will be used for simulation of the present three-car platoon using CFD.

\section{Numerical Setup and Discretization Scheme}

The three-dimensional flow governing equations were discretized and solved using a finite volume scheme, in a commercial CFD solver (STAR-CCM+). A segregated flow algorithm was employed, where the pressure-velocity equations were solved separately, and coupled using a predictor-corrector scheme. For temporal discretization, a more stable implicit scheme was used for time-marching the unsteady solution. Atmospheric conditions with velocity equivalent to the inlet velocity were defined as the initial conditions. A time step of $0.001 \mathrm{~s}$ was used in the simulation runs. In addition, air inlet velocity of $25 \mathrm{~m} / \mathrm{s}$ - equivalent to $90 \mathrm{~km} / \mathrm{h}$, which is a realistic velocity level for fuel savings on the road, was considered in all the platoon case studies.

Pressure forces were integrated from pressure distribution around the car surface and resolved to their $x$ and $y$ components to obtain respectively, the horizontal $($ drag $)$ and vertical (lift) forces. The drag coefficient $C_{D}=$ $\mathrm{drag} /\left(0.5 \rho U^{2} A\right)$ and lift coefficient $C_{L}=$ lift $/\left(0.5 \rho U^{2} A\right)$ were then determined based on the projected car frontal area $A$, which was estimated from the Ahmed body dimensions in Figure 2(b).

Overall, the computations were undertaken on a computer using 8GB RAM Intel i5 processor. The maximum run time in some of the cases took approximately 12 hours. 


\section{RESULTS AND DISCUSSION}

\section{Turbulence Model Study}

The objective of this study was to carry out computational fluid dynamic simulation involving different gaps between three cars. In addition, the second objective of the study was to identify the effect of the distance on drag and lift coefficients for each model of the car studied. The third objective is to identify the most suitable type of RANS turbulence model for simulation between $k-\varepsilon$ and $k$ - $\omega$ based on comparison with experiment results. Simulation results of one car and comparison with the study of Ahmed et al. [19] found that the turbulent $k$ - $\omega$ model yields the closest results to experimental value with $-0.56 \%$ error, compared to $k-\varepsilon$ with error of $0.88 \%$. The turbulent model study was further continued for a two-car platoon case and compared to experiments by Watkins and Vino [28]. Table 4 presents the comparison between the two turbulent models.

Table 4. Comparison of drag coefficients for two car platoon

\begin{tabular}{ccc}
\hline Model Type & $\begin{array}{c}\text { Drag coefficient } \\
\mathrm{C}_{\mathrm{D}}\end{array}$ & $\begin{array}{c}\text { Error } \\
\mathrm{C}_{\mathrm{D}}=0.25[28]\end{array}$ \\
\hline$k-\varepsilon$ & 0.317 & $21.14 \%$ \\
$k-\omega$ & 0.274 & $8.66 \%$ \\
\hline
\end{tabular}

Based on the table above, it can be seen that the turbulence $k$ - $\omega$ model once again results in a closer estimation to experimental value with an error of $8.66 \%$ compared to the $\mathrm{k}-\varepsilon$ with an error of $21.14 \%$. This may perhaps be due to the advantage of the SST (Menter) $k$ - $\omega$ model in comparison to the $k-\varepsilon$ model which may not be as accurate for near wall flow regions or flow with strong curvatures. Instead, the SST model behaves like a $k$ - $\omega$ model in near wall regions, allowing more accurate prediction for cases involving separating flows as in this car model. Thus, for the next simulation involving three cars, SST (Menter) $k$ - $\omega$ turbulence model will be used to find the drag and lift coefficients of each car. Figures 6 and 7 below illustrate the velocity streamlines for a single car and two car platoon case.

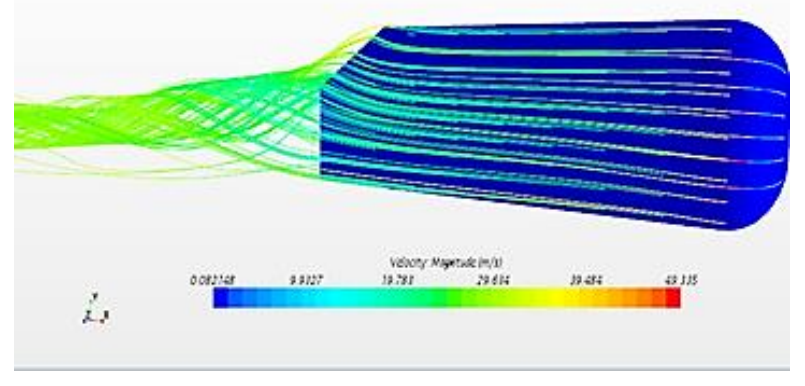

Figure 6. Velocity streamline for a single car (contours colored by velocity magnitude $(\mathrm{m} / \mathrm{s})$ )

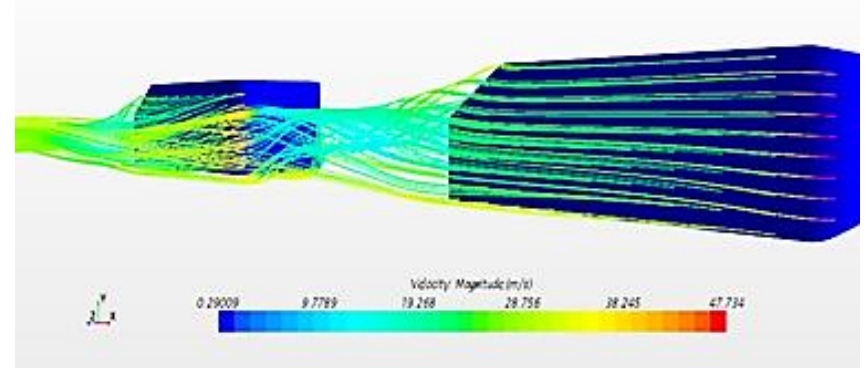

Figure 7. Velocity streamline of two cars (contours colored by velocity magnitude $(\mathrm{m} / \mathrm{s}))$

Based on the figure above, it can be seen in the case of a single car, the circulating flow known as the vortex is generated behind the car by the separation of the flow from the rear inclined angle of the car, as explained by Rajamani [13]. This strong vortex swirl contains high kinetic energy and reduces the rear pressure of the car, which then increases drag coefficient of the car. In the case of two car platoon, the presence of the second car resulted in the vortices generated from the rear of the first car to be disrupted, while reducing the vortex strength. As a result, the rear pressure of the first car is higher, yielding lower difference in pressure between the front and rear of the car and helped to reduce the drag coefficient of the car.

\section{Aerodynamic Forces}

In the case of three-car platoon, nine cases will be studied involving combination of distances between $0.5 \mathrm{~L}, 1.0 \mathrm{~L}$ and $1.5 \mathrm{~L} . X_{1}$ represents the distance between car 1 and 2 while the $X_{2}$ represents the distance between car 2 and 3 . Table 5 and Table 6 below show the results of the drag and lift coefficients generated by the simulation. 
Table 5. Drag coefficient values for three-car platoon

\begin{tabular}{cccccc}
\hline & Car Distance & Car Distance & \multicolumn{3}{c}{ Drag Coefficient } \\
\cline { 4 - 6 } Case & $\begin{array}{c}1 \& 2 \\
\left(X_{I}\right)\end{array}$ & $\begin{array}{c}2 \& 3 \\
\left(X_{2}\right)\end{array}$ & Car 1 & Car 2 & Car 3 \\
\hline 1 & $0.5 \mathrm{~L}$ & $0.5 \mathrm{~L}$ & 0.1217 & 0.2004 & 0.3408 \\
2 & $0.5 \mathrm{~L}$ & $1.0 \mathrm{~L}$ & 0.1242 & 0.2900 & 0.2740 \\
3 & $0.5 \mathrm{~L}$ & $1.5 \mathrm{~L}$ & 0.1244 & 0.3311 & 0.2630 \\
4 & $1.0 \mathrm{~L}$ & $0.5 \mathrm{~L}$ & 0.2179 & 0.1683 & 0.3100 \\
5 & $1.0 \mathrm{~L}$ & $1.0 \mathrm{~L}$ & 0.2252 & 0.2522 & 0.2856 \\
6 & $1.0 \mathrm{~L}$ & $1.5 \mathrm{~L}$ & 0.2244 & 0.2707 & 0.2670 \\
7 & $1.5 \mathrm{~L}$ & $0.5 \mathrm{~L}$ & 0.2257 & 0.1397 & 0.3152 \\
8 & $1.5 \mathrm{~L}$ & $1.0 \mathrm{~L}$ & 0.2546 & 0.2353 & 0.2934 \\
9 & $1.5 \mathrm{~L}$ & $1.5 \mathrm{~L}$ & 0.2493 & 0.2655 & 0.2734 \\
\hline
\end{tabular}

Table 6. The lift coefficient of three-car platoon

\begin{tabular}{c|c|c|c|c|c|}
\hline \multirow{2}{*}{ Case } & Car Distance & \multirow{2}{*}{\begin{tabular}{c} 
Car Distance \\
$1 \& 2$ \\
\cline { 4 - 6 }
\end{tabular}} & $\left(X_{I}\right)$ & $\begin{array}{c}2 \& 3 \\
\left(X_{2}\right)\end{array}$ & \multicolumn{3}{c}{$\begin{array}{c}\text { Lift Coefficient } \\
C_{L}\end{array}$} \\
\hline 1 & $0.5 \mathrm{~L}$ & $0.5 \mathrm{~L}$ & 0.0366 & 0.0560 & 0.1514 \\
\hline 2 & $0.5 \mathrm{~L}$ & $1.0 \mathrm{~L}$ & 0.0425 & 0.1829 & 0.0807 \\
\hline 3 & $0.5 \mathrm{~L}$ & $1.5 \mathrm{~L}$ & 0.0549 & 0.2738 & 0.0452 \\
\hline 4 & $1.0 \mathrm{~L}$ & $0.5 \mathrm{~L}$ & 0.0996 & -0.0078 & 0.0951 \\
\hline 5 & $1.0 \mathrm{~L}$ & $1.0 \mathrm{~L}$ & 0.1311 & 0.0800 & 0.1089 \\
\hline 6 & $1.0 \mathrm{~L}$ & $1.5 \mathrm{~L}$ & 0.1145 & 0.0377 & 0.0700 \\
\hline 7 & $1.5 \mathrm{~L}$ & $0.5 \mathrm{~L}$ & 0.1598 & -0.0611 & 0.1051 \\
\hline 8 & $1.5 \mathrm{~L}$ & $1.0 \mathrm{~L}$ & 0.1819 & $2.62 \mathrm{E}-8$ & 0.0959 \\
\hline 9 & $1.5 \mathrm{~L}$ & $1.5 \mathrm{~L}$ & 0.1641 & 0.1014 & 0.0466 \\
\hline
\end{tabular}

Based on the above table, it can be noted that cars that produce the minimum drag and lift coefficient values are different for each case. For example, in case 1, the drag coefficient value is the minimum for the first car (i.e. 0.1217). This is in contrast to case 4 where the drag coefficient value is the minimum for the second car (i.e. 0.1683). Similarly, for the lift coefficients, in which case the minimum value for case 1 is the first car (i.e. 0.0366), while for case 9, the minimum value is at the last car (i.e. 0.0466). In general, the different minimum values for each case are due to differences in the distance between vehicles for each case studied. Additionally, data also shows that when the same distance is applied between the three cars, the drag coefficient will increase in the order from the first car to the second car and the third car.

\section{Aerodynamic Trend between Cars}

Based on the table above, graphs for both drag and lift coefficients against car distance were plotted to obtain a clearer and more detailed view of how drag and lift coefficients vary by car distance. This part of the results will be divided into three main cases. The first case is when the distance between $X_{1}$ is set at $0.5 L$. The second case is when the distance of $X_{1}$ is $1.0 \mathrm{~L}$ and the third case when the distance of $X_{1}$ is set at $1.5 \mathrm{~L}$. For all three cases, the value of $X_{2}$ was varied between $0.5 \mathrm{~L}, 1.0 \mathrm{~L}$ and $1.5 \mathrm{~L}$, while drag and lift coefficient values were compared. Figures 8 and 9 respectively show graph of drag and lift coefficients against car distance, for the first case.

Based on Figure 8, it is found that for the first car, the drag coefficient does not vary much although the distance varies between the second and third cars. This is due to the first car drag coefficient being influenced mainly by its distance to the second car i.e. $0.5 \mathrm{~L}$. For the first car, the lowest drag coefficient is when the distance between cars 2 and 3 is $0.5 \mathrm{~L}$ (i.e. $C_{D}=0.1217$ ). For the second car, the lowest drag coefficient is also when $X_{2}=0.5 L$ between cars 2 and 3 , where $C_{D}$ $=0.2004$. While for the third car, the lowest drag coefficient is when $X_{2}=1.5 \mathrm{~L}$ between cars 2 and 3 (i.e. $C_{D}=$ 0.2630). Overall for this case, the lowest drag coefficient value is on the first car i.e. when $X_{1}=0.5 L$ between cars 1 and 2 , and $X_{2}=0.5 L$ between cars 2 and 3, where $C_{D}=0.1217$. 


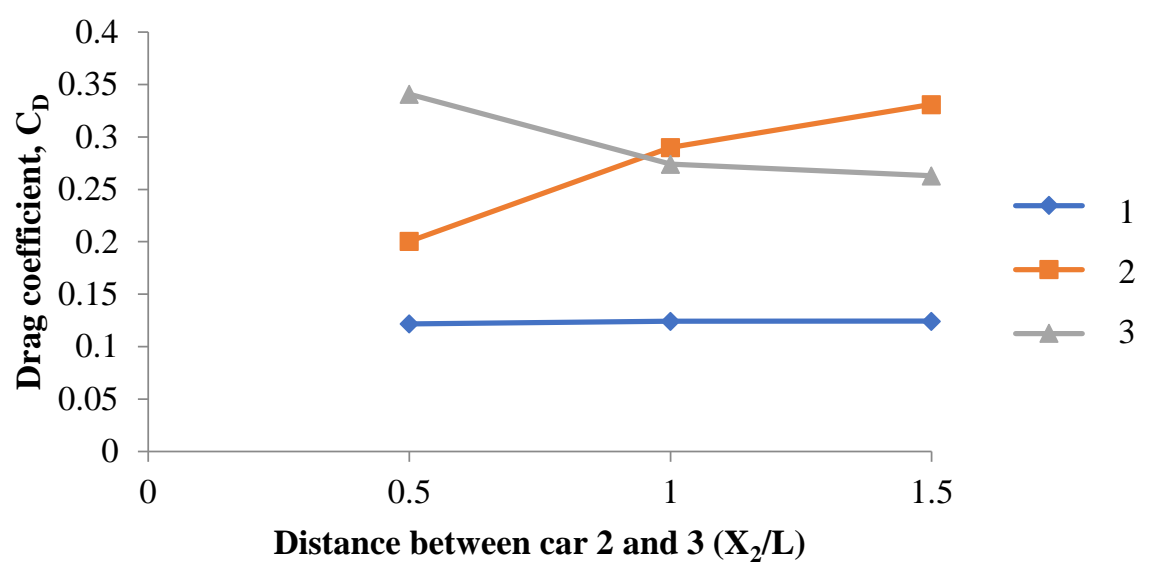

Figure 8. Graph of drag coefficient against car distance for the first case $\left(X_{1}=0.5 L, X_{2}=0.5 L, 1.0 L\right.$ and $\left.1.5 L\right)$

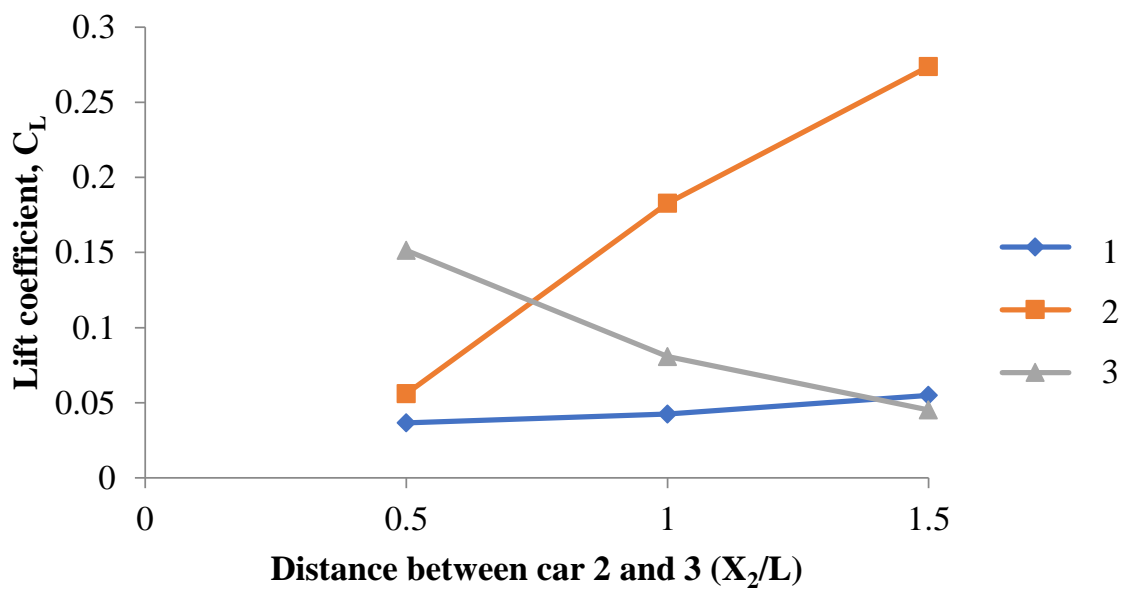

Figure 9. Graph of lift coefficient against the car distance for the first case $\left(X_{I}=0.5 \mathrm{~L}, X_{2}=0.5 \mathrm{~L}, 1.0 \mathrm{~L}\right.$ and $\left.1.5 \mathrm{~L}\right)$

Turning our attention to Figure 9, it is found that the same pattern as the drag coefficient can be seen in the lift coefficient. For all three cars, the coefficient of lift is the lowest for the same car distance as the drag coefficient, where $C_{L}$ is $0.0366,0.0560$ and 0.0452 . In addition, it was found in this case that the first car produced the lowest lift coefficient value when $X_{I}=0.5 \mathrm{~L}$ between cars 1 and 2 and $X_{2}=0.5 \mathrm{~L}$ between cars 2 and 3 . The study was then continued with the second case where the distance was extended to $1.0 L$ between cars 1 and 2. Figure 10 and Figure 11 respectively shows graph of the drag and lift coefficient versus distance between cars for the second case.

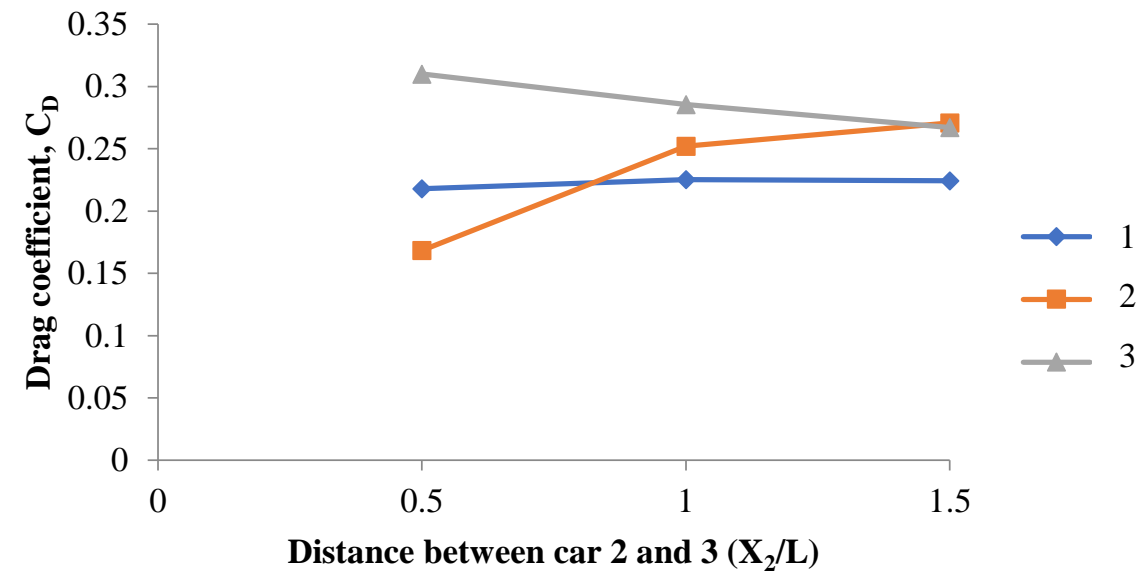

Figure 10. The drag coefficient graph against the car distance for the second case $\left(X_{I}=1.0 L, X_{2}=0.5 \mathrm{~L}, 1.0 \mathrm{~L}\right.$ and $\left.1.5 \mathrm{~L}\right)$ 
In this second case, it can be seen that when the distance is extended from $0.5 \mathrm{~L}$ for the first case to $1.0 \mathrm{~L}$ for the second case, the average drag coefficient of the first car increases. This is because when the distance between the cars goes farther, the vortex strength behind the first car is recovered (i.e. not disrupted by proximity of neighboring car) which reduces the back pressure of the first car while increasing its drag coefficient. This is verified by the velocity vector plots in Figure 16 and 17. A fully developed recirculation region can be seen (behind car 1) when car 2 is sufficiently far from car 1 , in comparison to the under-developed recirculation region when car 2 is $0.5 \mathrm{~L}$ away from car 1 , as shown in Figure 17 and 16 respectively. For the second car, just like in the first case, the lowest $C_{D}$ value is when the distance between cars 2 and 3 is $0.5 \mathrm{~L}$. Similarly, for the third car, the lowest $C_{D}$ value is when the gap between cars 2 and 3 is $1.5 L$. Overall, the lowest drag coefficient in this case is for the second car i.e. when car gap is $1.0 \mathrm{~L}$ between cars 1 and 2 , and $0.5 \mathrm{~L}$ gap between cars 2 and 3 (where $C_{D}=0.1683$ ).

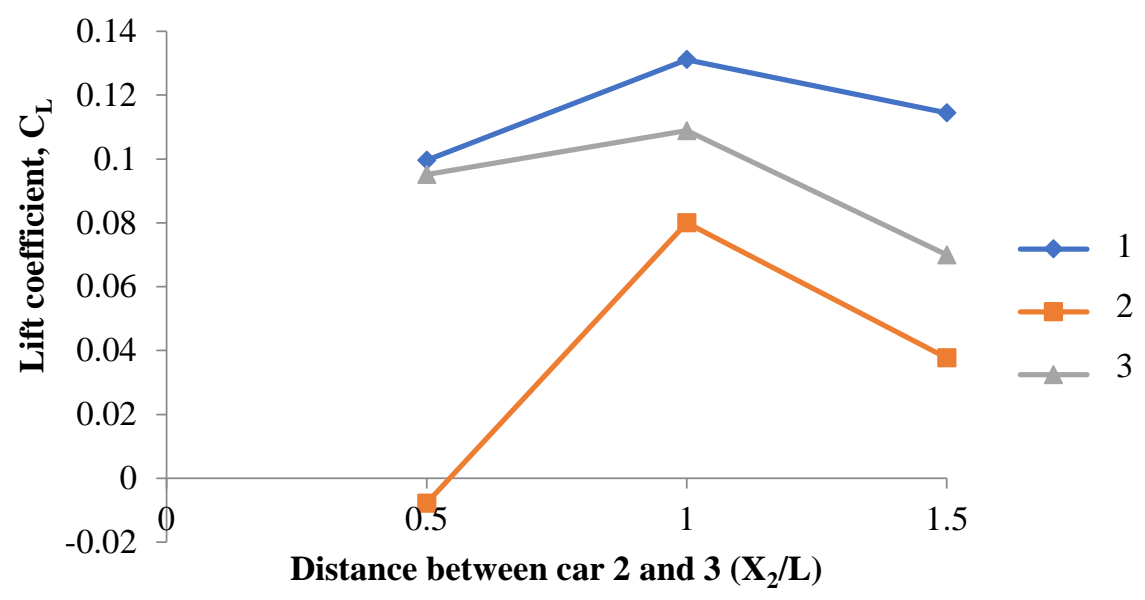

Figure 11. Graph of lift coefficient against car distance for the second case $\left(X_{I}=1.0 \mathrm{~L}, X_{2}=0.5 \mathrm{~L}, 1.0 \mathrm{~L}\right.$ and $\left.1.5 \mathrm{~L}\right)$

Based on Figure 11, significant differences are seen between the first, second and third car lift coefficients. On average, the second car clearly produces the lowest coefficient of lift compared to the first and third cars. The lowest lift coefficient in this case is on the second car with a distance of $1.0 L$ between cars 1 and 2 , and $0.5 L$ between cars 2 and 3 (i.e. $C_{L}=-0.0078$ ). The negative value in this coefficient of lift indicates that there is a change in the direction of lift to a force that push down the car rather than lifting the car upwards. This increases the attraction force between the car to the road surface without increasing the load of the car.

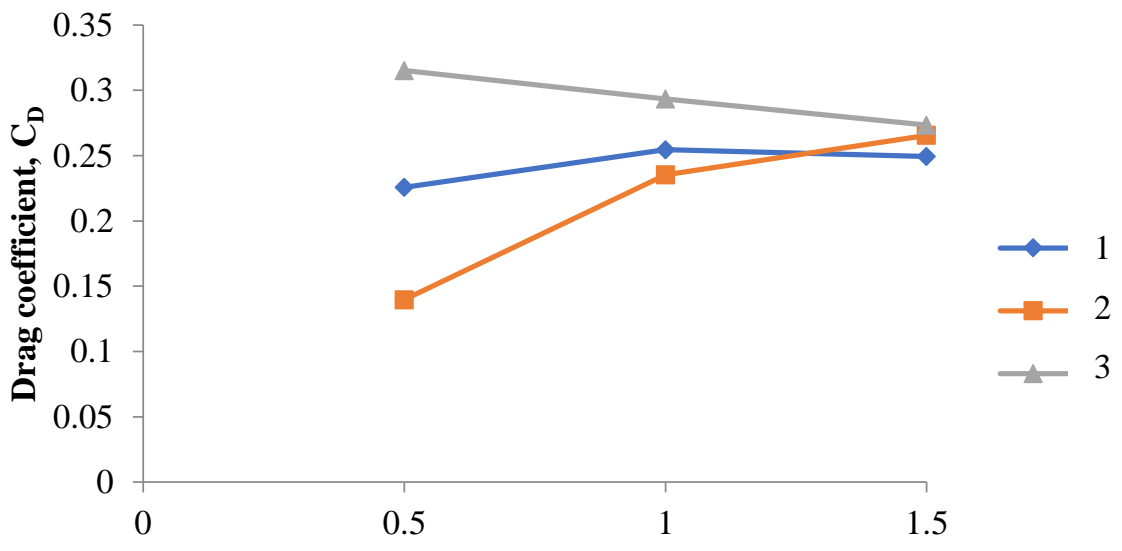

Distance between car 2 and $3\left(\mathrm{X}_{2} / \mathrm{L}\right)$

Figure 12. The graph of drag coefficient against car distance for the third case $\left(X_{l}=1.5 \mathrm{~L}, X_{2}=0.5 \mathrm{~L}, 1.0 \mathrm{~L}\right.$ and $\left.1.5 \mathrm{~L}\right)$

Figure 12 shows a drag coefficient graph against the distance between cars for the third case, where the spacing for cars 1 and 2 is fixed at $1.5 \mathrm{~L}$. It can be seen that the average drag coefficient for the first car is highest in this third case compared to its first and second cases. This proves the initial expectation of the study where, as the distance between cars 1 and 2 goes farther, the drag coefficient of the first car is increased and approaches the single car drag coefficient. Overall in this case, the lowest drag coefficient value is obtained for second car when $X_{1}=1.5 L$ between cars 1 and 2 , and $X_{2}=$ $0.5 L$ between cars 2 and 3 (i.e. $C_{D}=0.1397$ ). 


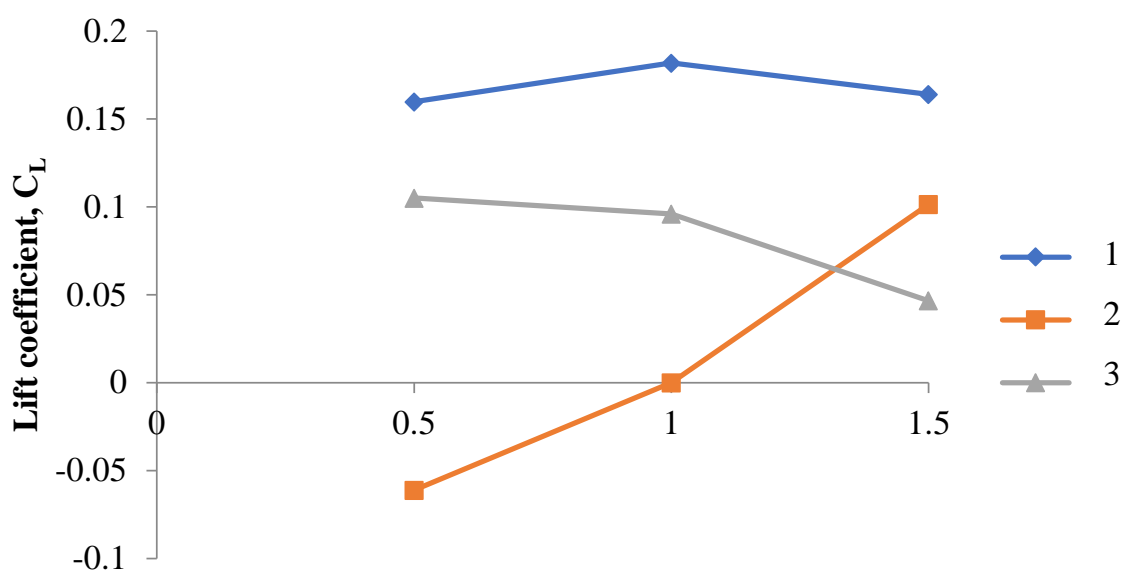

Distance between car 2 and $3\left(\mathrm{X}_{2} / \mathrm{L}\right)$

Figure 13. Graph of lift coefficient against car distance car for third case $\left(X_{l}=1.5 \mathrm{~L}, X_{2}=0.5 \mathrm{~L}, 1.0 \mathrm{~L}\right.$ and $\left.1.5 \mathrm{~L}\right)$

Based on Figure 13, there are different patterns between the first, second and third cars. For the first car, the average coefficient of the lift is the highest compared to the second and third cars. The second and third car shows an upward and downward pattern respectively, for their lift coefficient when the distance between cars 2 and 3 is increased. In addition, for this third case $\left(X_{1}=1.5 \mathrm{~L}\right.$ between cars 1 and 2$)$, the lowest lift coefficient value is in the second car when $X_{2}=0.5 \mathrm{~L}$ between cars 2 and 3 -i.e. $C_{L}=0.0611$. Overall, the car that yields the lowest drag or lift coefficients varies for each case of the distance being studied. There appears a link between the two aerodynamic values where the car that produces the lowest drag coefficient also yields the lowest lift coefficient.

\section{Fluid Flow Visualization}

Figure 14 depicts velocity streamline on car platoon that produces the lowest drag and lift coefficients on the first car, where $C_{D}=0.1217$ for the first car, 0.2004 for the second car and 0.3408 for the third car. The distance between cars 1 and 2 and 2 and 3 is equal to $0.5 \mathrm{~L}$. Figure 14 shows the difference in the vortex behind the cars, where for the first car, the vortex is weaker and could not be formed compared to behind the second and third cars. This also reduces the kinetic energy behind the first car. The results can be seen with the low streamline velocity recorded behind the first car. This low velocity increases the rear pressure of the first car, which reduces the drag coefficient of the car. For platoon cases producing the lowest drag coefficient on the second car, it can be seen clearly for $X_{1}=1.5 \mathrm{~L}$ distance between cars 1 and 2 , and $X_{2}=0.5 L$ between cars 2 and 3 . Figure 15 below shows the velocity streamline of this platoon case.

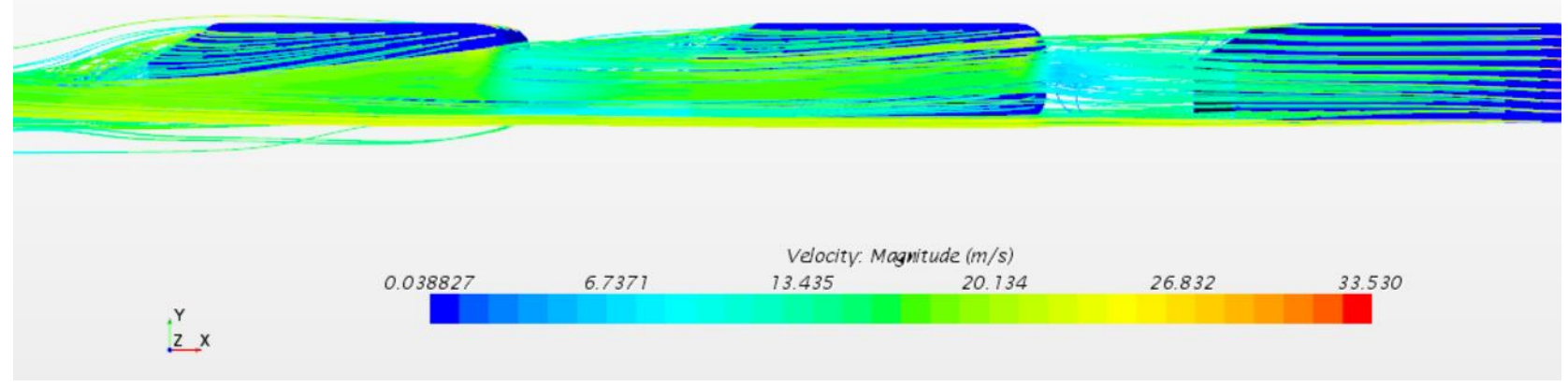

Figure 14. Car velocity streamline $\left(X_{1}=0.5 L, X_{2}=0.5 L\right)$ 


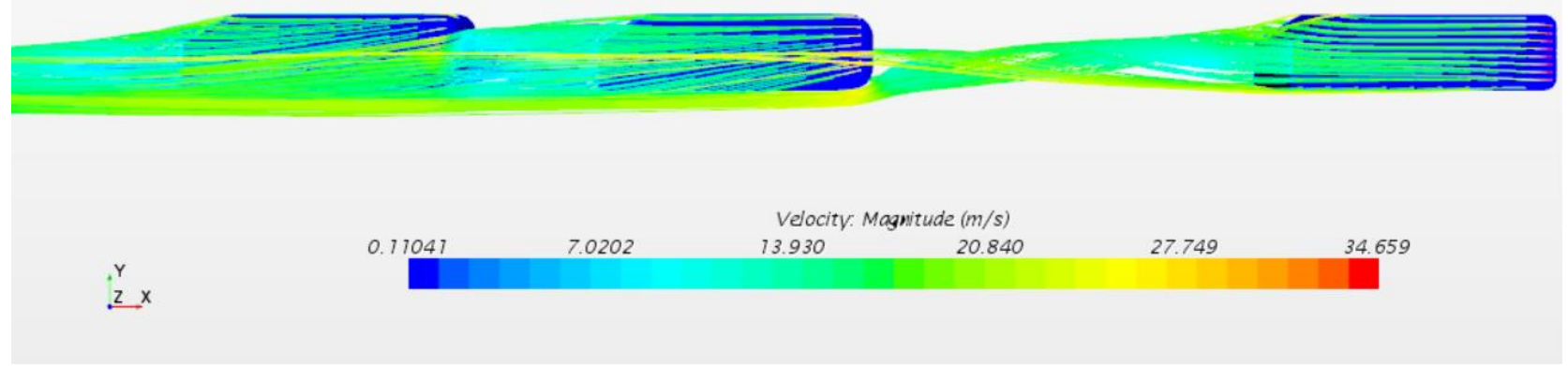

Figure 15. Car velocity streamline $\left(X_{l}=1.5 L, X 2=0.5 L\right)$

Based on Figure 15 above, the difference in vortex formed behind the three cars could be observed. In this case, the second car recorded the lowest drag coefficient of 0.1397 in comparison to 0.2257 for the first car and 0.3152 for the third car. From the resulting streamline, for the first car, the distance with the second car of $1.5 \mathrm{~L}$ causes no effective interference in the production of vortex swirls behind the first car, thereby increasing the rotary kinetic energy while reducing the vehicle's back pressure and raising the drag coefficient. The same can be seen on the last car on this train where no backto-back car interfering with its vortex, causing a high drag coefficient. Meanwhile, for the second car, the presence of a third rear car at a distance of $0.5 \mathrm{~L}$ disrupts the production of vortex swirls, which reduces the resulting kinetic energy, while increasing the rear pressure of the car and reducing its drag coefficient. The rear velocity of the second car also indicates low velocity region corresponding to high rear pressure compared to rear regions of other cars.

The car lift coefficient is closely related to the attractive force of the car to the road surface. When the force is positive, the force will be directed upwards and reduces the stability of the car, while when it is negative, the force will act downward and increase the stability and attraction of the car to the surface. Figure 16 shows the velocity contour resulting in the case where the lowest drag coefficient is on the first car.

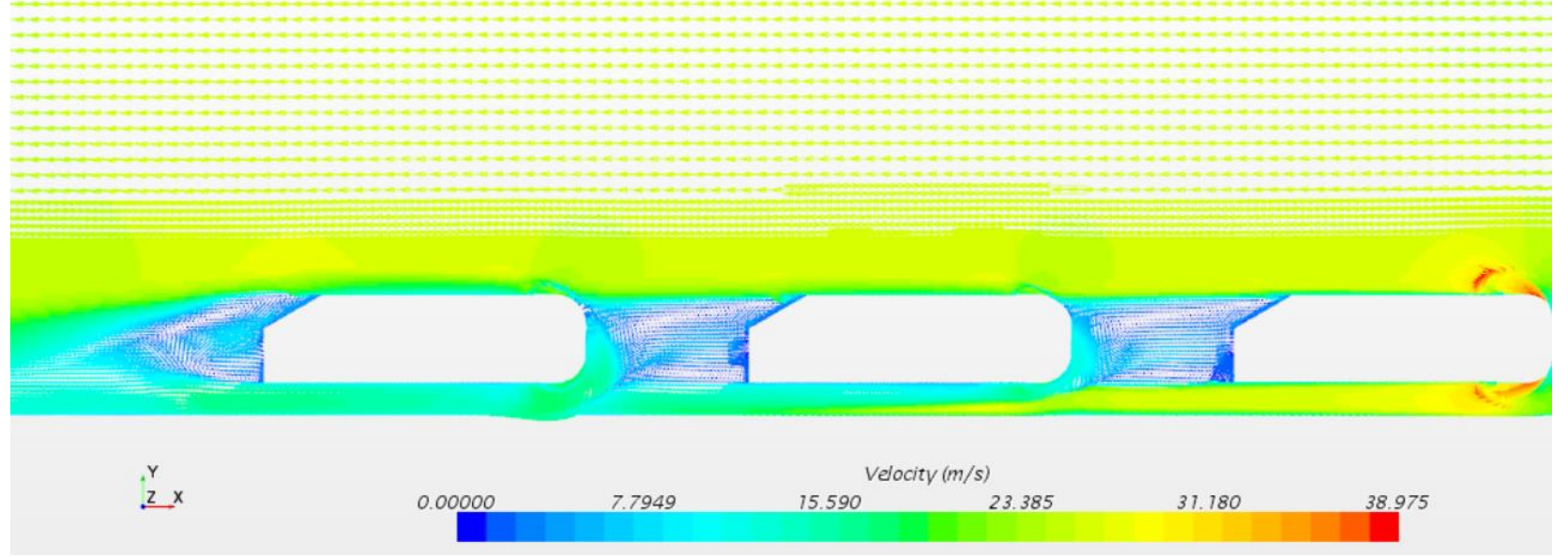

Figure 16. Car velocity vector $\left(X_{I}=0.5 L, X_{2}=0.5 L\right)$

Figure 16 shows the car velocity vector for distance equals $0.5 \mathrm{~L}$ between cars 1 and 2 , and cars 2 and 3 . In this case, the coefficient of lift for the first car is lowest with $C_{L}=0.0366$ compared to 0.0560 for second car and 0.1514 for third car. It could be observed from the flow velocity that in the first car, its velocity below the car is highest compared to the second and third cars. Based on the principle of Bernoulli, with high velocity, the value of pressure under the car will decrease, thereby reducing its lift coefficient and improving the stability of the car on the road. Figure 17 below shows the platoon case that produces negative lift coefficient on the second car. 


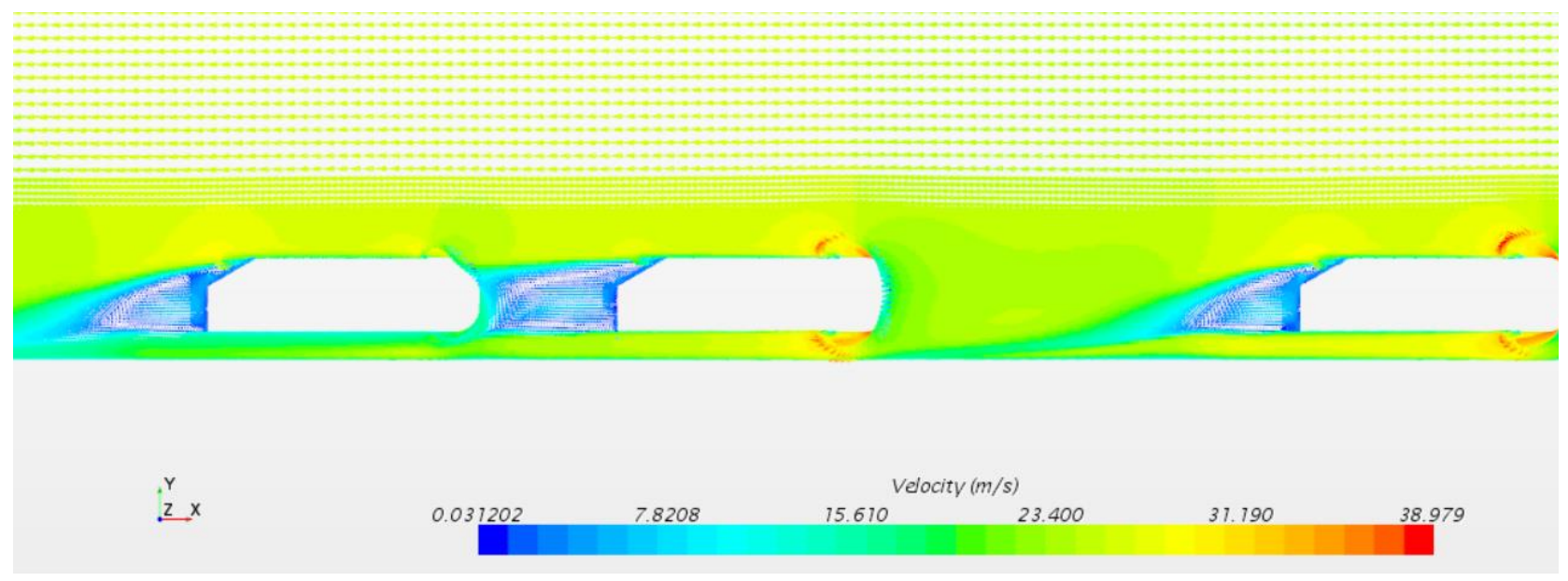

Figure 17. Car velocity vector $\left(X_{1}=1.5 L, X_{2}=0.5 L\right)$

In the case where $X_{I}=1.5 \mathrm{~L}$, the value of the lift coefficient for first of the three cars is not much different when $X_{2}$ is varied. However, the negative lift coefficient that results in the second car when $X_{2}=0.5 \mathrm{~L}$ indicates a change in the direction of the its lift from pushing upwards to pushing downwards. Based on Figure 17 above, this phenomenon may be explained by high flow velocities below the car resulting in significant pressure drop underneath and reduced drag coefficients, in addition to the proximity of the car behind that assists aerodynamic efficiency of the second car. This downforce generate traction between the car and the road surface while improving the stability of the car.

\section{CONCLUSIONS}

Based on the simulation conducted and comparison with the single car experimental study by Ahmed et al. [19], it was found that the turbulent $k$ - $\omega$ approximate the experimental value with error of $-0.56 \%$ compared to the $k-\varepsilon$ model which had an error of $0.88 \%$. The next comparison of two Ahmed car with experiments by Watkins and Vino [28] found that the $k$ - $\omega$ model was more accurate (with error of $8.66 \%$ ) compared to $k-\varepsilon$ model with a high error of $21.14 \%$. This study has also successfully undertaken a computational fluid dynamic simulation involving different gaps for three Ahmed cars in a platoon. A total of 9 gap cases were investigated between cars 1 and $2\left(X_{I}\right)$ and cars 2 and $3\left(X_{2}\right)$ involving $0.5 \mathrm{~L}, 1.0 \mathrm{~L}$ and $1.5 \mathrm{~L}$ distances. It was found that the lowest drag coefficient that impacts on vehicle fuel savings varied depending on car positions. It can be concluded that the for lead car ahead, the lowest drag coefficient may be observed for a distance $X_{1}=0.5 L$ and $X_{2}=0.5 L$ (where $C_{D}=0.1217$ ). The lift coefficient for this car is 0.0366 . For the middle car, the lowest drag coefficient occurs at distance $X_{I}=1.5 \mathrm{~L}$ and $X_{2}=0.5 \mathrm{~L}$, where $C_{D}$ is 0.1397 . The lift coefficient on this car is - 0.0611 . Meanwhile for the last car, the lowest drag coefficient may be seen when $X_{1}=0.5 L$ and $X_{2}=1.5 L$, i.e. $C_{D}=$ 0.2630 . The coefficient of lift for this car is 0.0452 . Drag coefficients were also found to decline from front car to rear car when different gaps are set between three cars and have a positive impact on fuel savings for cars in a platoon.

\section{ACKNOWLEDGMENTS}

The authors gratefully acknowledge support from Ministry of Higher Education (Malaysia) under research grants FRGS/1/2018/TK03/UKM/02/5.

\section{REFERENCES}

[1] Y. Ko, B. Song, and Y. Oh, "Mathematical analysis of environmental effects of forming a platoon of smart vehicles," Sustainability, vol. 11, no. 3, p. 571, 2019, doi: 10.3390/su11030571.

[2] S. Maiti, S. Winter, and L. Kulik, "A conceptualization of vehicle platoons and platoon operations," Transp. Res. Part C Emerg. Technol., vol. 80, pp. 1-19, 2017, doi: 10.1016/j.trc.2017.04.005.

[3] W. Zhang, M. Sundberg, and A. Karlström, "Platoon coordination with time windows: an operational perspective," Transp. Res. Procedia, vol. 27, pp. 357-364, 2017, doi: 10.1016/j.trpro.2017.12.129.

[4] R. Horowitz, S. Sastry, and P. . Varaiya, "Hybrid supervisory control for modes of operation," in Automated Vehicle and Highway Systems (AVHS), 1998.

[5] A. Davila, E. del Pozo, E. Aramburu, and A. Freixas, "Environmental benefits of vehicle platooning," SAE Technical Paper, 2013, doi: 10.4271/2013-26-0142.

[6] L. Zhang, F. Chen, X. Ma, and X. Pan, "Fuel economy in truck platooning: A literature overview and directions for future research," J. Adv. Transp., vol. 2020, pp. 1-10, 2020, doi: 10.1155/2020/2604012. 
[7] Z.-F. Yang, S.-H. Li, A.-M. Liu, Z. Yu, H.-J. Zeng, and S.-W. Li, "Simulation study on energy saving of passenger car platoons based on DrivAer model," Energy Sources, Part A Recover. Util. Environ. Eff., vol. 41, no. 24, pp. 3076-3084, 2019, doi: 10.1080/15567036.2019.1587050.

[8] W.-H. Hucho, "Aerodynamics of road vehicles: From fluid mechanics to vehicle engineering," in Aerodynamics of Road Vehicles, W. Hucho, Ed. Warrendale, PA: Society of Automotive Engineers, 1998, pp. 13-26, 49-51.

[9] M. A. Siemon, "Numerical analysis of heterogeneous and homogeneous truck platoon aerodynamic drag reduction," MSc Thesis: Auburn University, 2018.

[10] R. Gnatowska and M. Sosnowski, "The influence of distance between vehicles in platoon on aerodynamic parameters," $E P J$ Web Conf., vol. 180, p. 2030, 2018, doi: 10.1051/epjconf/201818002030.

[11] F. H. Robertson et al., "An experimental investigation of the aerodynamic flows created by lorries travelling in a long platoon," J. Wind Eng. Ind. Aerodyn., vol. 193, p. 103966, 2019, doi: 10.1016/j.jweia.2019.103966.

[12] F. Browand, J. McArthur, and C. Radovich, "Fuel saving achieved in the field test of two tandem trucks, PATH Research Report UCB-ITS-PRR-2004-20," 2004.

[13] G. Rajamani, "CFD analysis of air flow interactions in vehicle platoons," Postgraduate Thesis: RMIT, Australia, 2006.

[14] P. Schito and F. Braghin, "Numerical and experimental investigation on vehicles in platoon," SAE Int. J. Commer. Veh., vol. 5, no. 1, pp. 63-71, 2012, doi: 10.4271/2012-01-0175.

[15] B. Daryakenari, S. Abdullah, R. Zulkifli, E. Sundararajan, and A. B. M. Sood, "Numerical study of flow over Ahmed body and a road vehicle and the change in Aerodynamic characteristics caused by rear spoiler," Int. J. Fluid Mech. Res., vol. 40, no. 4, pp. 354-372, 2013, doi: 10.1615/interjfluidmechres.v40.i4.50.

[16] V. L. Knoop, M. Wang, I. Wilmink, D. M. Hoedemaeker, M. Maaskant, and E.-J. Van der Meer, "Platoon of SAE level-2 automated vehicles on public roads: Setup, traffic interactions, and stability," Transp. Res. Rec. J. Transp. Res. Board, vol. 2673, no. 9, pp. 311-322, 2019, doi: 10.1177/0361198119845885.

[17] M. Norouzi, and M.A. Pooladi, M. Mahmoudi, and and, "Numerical investigation of drag reduction in a Class 5 medium duty truck," J. Mech. Eng. Sci., vol. 10, no. 3, pp. 2387-2400, 2016, doi: 10.15282/jmes.10.3.2016.15.0221.

[18] T. Han, "Computational analysis of three-dimensional turbulent flow around a bluff body in ground proximity," AIAA J., vol. 27, no. 9, pp. 1213-1219, 1989, doi: 10.2514/3.10248.

[19] S. R. Ahmed, G. Ramm, and G. Faltin, "Some salient features of the time-averaged ground vehicle wake," SAE Paper 840300, 1984, doi: $10.4271 / 840300$.

[20] C. Hinterberger, M. Garcia-Villalba, and W. Rodi, "Large eddy simulation of flow around the Ahmed body," in The Aerodynamics of Heavy Vehicles: Trucks, Buses, and Trains, R. McCallen, F. Browand, and J. Ross, Eds. Springer Berlin Heidelberg, 2004, pp. 77-87.

[21] R. S. Khan and and Sudhakar Umale, "CFD aerodynamic analysis of Ahmed body," Int. J. Eng. Trends Technol., vol. 18, no. 7, pp. 301-308, 2014, doi: 10.14445/22315381/ijett-v18p262.

[22] I. Bayraktar, D. Landman, and O. Baysal, "Experimental and computational investigation of Ahmed body for ground vehicle aerodynamics," SAE Technical Paper 2001-01-2742, 2001, doi: 10.4271/2001-01-2742.

[23] M. Minguez, R. Pasquetti, and E. Serre, "High-order large-eddy simulation of flow over the 'Ahmed body' car model," Phys. Fluids, vol. 20, no. 9, p. 95101, 2008, doi: 10.1063/1.2952595.

[24] C.-H. Bruneau, E. Creusé, D. Depeyras, P. Gilliéron, and I. Mortazavi, "Coupling active and passive techniques to control the flow past the square back Ahmed body," Comput. Fluids, vol. 39, no. 10, pp. 1875-1892, 2010, doi: 10.1016/j.compfluid.2010.06.019.

[25] E. Serre et al., "On simulating the turbulent flow around the Ahmed body: A French-German collaborative evaluation of LES and DES," Comput. Fluids, vol. 78, pp. 10-23, 2013, doi: 10.1016/j.compfluid.2011.05.017.

[26] P. Madharia, M. . Tiwari, and K. Ravi, "Computational simulation of Ahmed body with varying nose radius, Ground Height \& Rear Slant angle,” Int. J. Res. Appl. Sci. Eng. Technol., vol. 3, no. 5, pp. 925-932, 2015.

[27] C.-K. Choi and D.-K. Kwon, "Wind tunnel blockage effects on aerodynamic behavior of bluff body," Wind Struct., vol. 1, no. 4, pp. 351-364, 1998, doi: 10.12989/was.1998.1.4.351.

[28] S. Watkins and G. Vino, "The effect of vehicle spacing on the aerodynamics of a representative car shape," J. Wind Eng. Ind. Aerodyn., vol. 96, no. 6-7, pp. 1232-1239, 2008, doi: 10.1016/j.jweia.2007.06.042.

[29] T.-H. Shih, W. W. Liou, A. Shabbir, Z. Yang, and J. Zhu, "A new k-€ eddy viscosity model for high reynolds number turbulent flows," Comput. Fluids, vol. 24, no. 3, pp. 227-238, 1995, doi: 10.1016/0045-7930(94)00032-t. 